(2) Open Access Full Text Article

REVIEW

\title{
Resveratrol As A Natural Regulator Of Autophagy For Prevention And Treatment Of Cancer
}

This article was published in the following Dove Press journal: OncoTargets and Therapy

Yuanyuan Tian'

Wenjing Song'

Dan $\mathrm{Li}^{1}$

Lu Cai ${ }^{2}$

Yuguang Zhao'

'Cancer Center, the First Hospital of jilin University, Changchun, Jilin I3002I, People's Republic of China;

${ }^{2}$ Departments of Pediatrics, Radiation Oncology, Pharmacology and Toxicology, University of Louisville, Louisville, KY 40292, USA
Correspondence: Yuguang Zhao

Cancer Center, the First Hospital of Jilin University, Changchun, Jilin I3002I,

People's Republic of China

$\mathrm{Tel}+8643$ I I5804303822

Email zhaoyuguang@jlu.edu.cn

\begin{abstract}
Resveratrol, as a natural product compound, has been recently attracted much attention for its potent effects on cancer. Cancer is a serious disease threatening human survival and social development. Autophagy is a cellular pathway to realize the metabolic needs of the cell itself and the renewal of some organelles and plays opposing, contextdependent role in tumorigenesis. So the regulation of autophagy is of great significance in the treatment of cancer. p62, as an autophagy adaptor protein, is a preferred target for autophagy and is constantly controlled by constitutive autophagy. As a tumor-suppression mechanism, autophagy deficiency is common in tumors, which results in aberrant accumulation of p62 and activates p62-regulated pathways, such as activation of mTOR in nutrient sensing, and the activation of the Keap1-Nrf2 pathway for antioxidant stress, which are associated with cancer development. In this review, we emphasize that resveratrol can induce autophagy in the treatment of cancer and accelerates the degradation of p62, and then, the mTOR activation is blocked and Nrf2 activation is suppressed. As a result, the multidrug resistance of cancer cells can be reversed by resveratrol.
\end{abstract}

Keywords: resveratrol, cancer, autophagy, p62, mTOR, Nrf2

\section{Introduction}

Cancer, as a growing health problem worldwide, affects millions of people every year. According to International Agency for Research on Cancer (IARC), the global cancer burden is increasing. If no effective measures were taken, it is expected that by 2030 , there will be 22 million new cancer cases a year and the number of cancer deaths will rise to 13 million during the same period. ${ }^{1}$ Cancer will transcend cardiovascular disease and become the most important health problem in the $21 \mathrm{st}$ century. It will seriously endanger the survival of human and bring heavy burden to the economic development. Although certain achievements were made in the continuous struggle with cancer, it is still a long way to go to conquer cancer. Current cancer therapies, such as chemotherapy, radiotherapy, molecular targeted therapy, and immune checkpoint inhibitor, are limited by the development of resistance to these therapies. ${ }^{2}$ Therefore, we need to identify a new strategy or new therapeutic agent that can overcome resistance. Because of their safety and low toxicity, natural compounds have gained much attention in the prevention and treatment of cancer. Resveratrol, as a naturally occurring stilbene phytoestrogen, mainly found in many foods, such as mulberry, peanut, pineapple, grape, and wine, 
Table I The Mechanisms Of Resveratrol Inducing Autophagy In Different Cancers

\begin{tabular}{|c|c|c|c|}
\hline Cancer Type & The Mechanism Of Resveratrol Effect & Findings & References \\
\hline Lung cancer & $\begin{array}{l}\text { Mediate autophagocytic death through Ca2 } \\
\text { +/AMPK-mTOR signaling pathway }\end{array}$ & Inducing growth inhibition and cell death & 23 \\
\hline Ovarian cancer & $\begin{array}{l}\text { Inducing reactive ROS generation } \\
\text { Inactivating STAT3 signaling, upregulating two } \\
\text { autophagy-related genes, LC3 and Beclin-I }\end{array}$ & $\begin{array}{l}\text { Enhancing apoptotic and autophagic activities } \\
\text { Increasing sensitivity to chemotherapy drugs }\end{array}$ & $\begin{array}{l}25 \\
81\end{array}$ \\
\hline Oral cancer & $\begin{array}{l}\text { Inducing autophagy and apoptosis by stimulating } \\
\text { the expression of autophagic protein }\end{array}$ & $\begin{array}{l}\text { Inducing autophagic pathway switched to the apoptotic } \\
\text { machinery, and improving the sensitivity to cisplatin }\end{array}$ & 5 \\
\hline $\begin{array}{l}\text { Colorectal } \\
\text { carcinomas }\end{array}$ & $\begin{array}{l}\text { Inducing autophagy and initiating Caspase-8/ } \\
\text { Caspase-3-dependent apoptosis via producing } \\
\text { ROS }\end{array}$ & Suppressing the growth of cancer cells & 82 \\
\hline Prostate carcinomas & $\begin{array}{l}\text { Activating autophagic cell death via } \\
\text { downregulating STIMI and mTOR pathway }\end{array}$ & Inhibiting cell proliferation and activating apoptosis & 83 \\
\hline Breast carcinomas & $\begin{array}{l}\text { Increasing PTEN protein levels and decreasing } \\
\text { phosphorylation of AKT }\end{array}$ & $\begin{array}{l}\text { Resensitizing cancer cells to treatments and inducing } \\
\text { apoptosis and inhibiting cell proliferation }\end{array}$ & 84 \\
\hline $\begin{array}{l}\text { Head and neck } \\
\text { cancer }\end{array}$ & $\begin{array}{l}\text { Inhibiting the Nrf2/ARE pathway and facilitating } \\
\text { p62 degradation }\end{array}$ & Reversing the resistance to ferroptosis in $\mathrm{HNC}$ cells & 73 \\
\hline
\end{tabular}

exhibits chemopreventive and therapeutic effects on several cancers by targeting some molecules that play important roles in tumourigenesis. ${ }^{3,4}$ Studies have shown that resveratrol can induce autophagy in cancer cells. ${ }^{5}$ Autophagy is primarily a mechanism that suppresses tumorigenesis by maintaining genome integrity and preventing proliferation and inflammation. ${ }^{6}$ Allelic loss beclin 1 or constitutive activation of the autophagy-suppressing pathway such as phosphatidylinositol 3-kinase (PI3K)/ Mammalian Target of Rapamycin (mTOR) pathway are common causes of autophagy defects in cancer cells. ${ }^{7}$ Therefore, autophagy regulation plays an important role in cancer therapies, and the development of efficient approaches to modulate the function of autophagy is critical. In this review, we describe how the autophagy pathway plays an important role during cancer development and emphasize that resveratrol can induce autophagy via mTOR signaling pathway and accelerate the degradation of p62 in the treatment of cancer, and further discuss the effect of resveratrol on autophagy and apoptosis in different cancers. We also illustrate the cytotoxic enhancement effect of resveratrol and its synergy with other chemotherapeutic agents and autophagy inhibitors.

\section{Autophagy And Cancer}

Autophagy is a process of degrading cytoplasmic proteins or organelles, which are first wrapped in vesicles and then fused with lysosomes to form autophagy lysosomes, and finally degraded in autophagy lysosomes. In this process, the metabolic needs of the cell itself and the renewal of some organelles are realized. ${ }^{8}$ As a major cellular route for degrading its own cytoplasmic long-lived proteins and cytoplasmic organelles, this kind of lysosome exists widely in normal cells and acts as a "scavenger" in the cells, which acts as a normal pathway for the natural reduction and renewal of intracellular organelles and other structures. Autophagic lysosomes greatly increase when tissue cells are injured by various physical and chemical factors, so they play a protective role in cell injury and malignant transformation. Although autophagy has been shown to suppress tumorigenesis, its effect is context-dependent. Once a tumor occurs, tumor cells can use this process to survive in hostile metabolic microenvironments and use autophagic substrates to maintain tumor growth. ${ }^{6}$ The discovery of the ATG (autophagyrelated proteins) gene beclin 1, as a candidate tumor suppressor gene in human breast carcinoma, was the first specific link between the autophagy mechanism and human cancer. ${ }^{9}$ It has been reported that the critical autophagy gene beclin 1 is mono-allelically lost in $40 \%$ to $75 \%$ of the human prostate, breast, and ovarian cancers, which shows that autophagy plays an important role in tumorsuppression mechanism. ${ }^{9-11}$ Over the past decade, some of the genetic correlations that exist between autophagy defects and cancer development have been the basis for the theory that autophagy is the real tumor-suppressing pathway. ${ }^{12,13}$ Recent studies have shown that several proteins and signaling pathways associated with autophagy, 
such as PI3K/Akt, Ras, and Myc oncogenes, are involved in tumorigenesis and lead to the inhibition of autophagy. ${ }^{14}$ There are some signaling pathways regulating both autophagy and tumorigenesis. The tumor suppressor genes, such as PTEN, tuberous sclerosis complex 1 (TSC1), and tuberous sclerosis complex 2 (TSC2) can inhibit the upstream signal of mTOR and stimulate autophagy, while PI3K/Akt inhibits autophagy by activating mTOR signaling pathway. ${ }^{15}$ In this review, we focus on autophagy induced by resveratrol through the regulation of mTOR and p62 signaling pathway.

\section{Resveratrol - An Inducer Of Autophagy \\ Anticancer Effects Of Resveratrol Combined With Different Polyphenols}

Resveratrol (3,5,4-trihydroxystilbene) is a naturally occurring non-flavonoid polyphenol compound, which is an antitoxin produced by many plants under various external stimuli, such as excessive sunlight, ultraviolet radiation, and invading fungal infection. ${ }^{16}$ Resveratrol is found in various food such as grape, mulberry, cranberry, and peanut with different concentrations. ${ }^{17}$ And its cancer-preventive and anti-cancer properties have attracted considerable attention in the last two decades. ${ }^{18}$ The first real interest in the cancer chemopreventive activity of resveratrol was reported by Jang and colleagues that resveratrol has the ability to inhibit cellular events associated with tumorigenesis and progression. ${ }^{19}$ Epidemiological research shows that women who eat grapes rich in resveratrol have a reduced risk of developing breast cancer by at least $50 \% .{ }^{20}$ Resveratrol can act on signal-transduction pathways associated with the initiation and progression of cancer, such as pathways that affect inflammation, angiogenesis, cell growth and division, apoptosis, and metastasis. ${ }^{2}$ It has been demonstrated that resveratrol exhibits cytotoxicity and concentration-dependently suppresses cell viability in prostate cancer, oral cancer, non-small cell lung cancer, but has low toxicity in normal cells. 5,21,22 Many compounds derived from natural products, such as quercetin and curcumin, also can act against cancer by inducing autophagy. The combination of different polyphenols may elicit synergistic or antagonistic effects. ${ }^{23}$ In previous studies, it was argued that the combination of curcumin and resveratrol had synergistic anti-cancer effects in breast cancer, colon cancer, hepatocellular cancer, and lung cancer. $^{24-27}$ Surprisingly, despite both resveratrol and quercetin activated the autophagy process, the combination of them resulted in an antagonistic effect in the human hepatoblastoma HepG2 cells. $^{28}$

\section{The Effect Of Resveratrol On Autophagy And Apoptosis In Different Cancers}

Apoptosis and autophagy are two self-destructive processes. $^{7,29}$ The functional relationship between apoptosis and autophagy is complex, and in some cases, autophagy forms a stress adaptation that protects cells from apoptosis, while in other cell environments, autophagy can induce cell death by itself or initiate apoptosis. ${ }^{30}$ Here, we discuss the effect of resveratrol on autophagy and apoptosis in different cancers (Table 1). It has been shown that resveratrol could mediate autophagocytic death through $\mathrm{Ca}^{2+} / \mathrm{AMPK}-\mathrm{mTOR}$ signaling pathway, and treatment human non-small cell lung cancer cells (A549) with resveratrol could also induce cell death through autophagy via this signaling pathway, but not apoptosis. $^{31}$ Lang and colleagues reported that resveratrol could induce cell death via inducing reactive oxygen species (ROS) generation and triggering autophagy and apoptosis in human ovarian cancer (OVCAR-3) cells, and this effect can be significantly attenuated by the autophagy inhibitor chloroquine. ${ }^{32}$ It also has been proved that resveratrol can induce autophagy in SKOV3 human ovarian cancer cells and inhibit apoptosis. However, when autophagy inhibitor 3-methyladenine (3-MA), an inhibitor of PI3K, is applied simultaneously, resveratrol efficiently promotes cell apoptosis through inhibiting autophagy, and the inhibition effect on cell survival is significantly stronger, which suggests that autophagy induced by resveratrol may be protective for SKOV3 cells as a protective autophagy. ${ }^{33}$ Moreover, it has been shown that resveratrol can induce autophagy and apoptosis by stimulating the expression of autophagic protein in cisplatin-resistant human oral cancer CAR cells. It also demonstrated that accumulation of autophagic vacuoles was before pronounced hallmarks of apoptosis appeared, suggesting that the autophagic pathway switched to the apoptotic machinery in the process of resveratrol inducing CAR cell death. It also has been proved that 3-MA can specifically block the fusion of autophagosomes with lysosome and enhanced CAR cell viability after treatment with resveratrol. ${ }^{5}$

\section{Anticancer Effects Of Resveratrol Combined With Chemotherapeutic Drug} Chemotherapy, as the principal treatment of cancer, drug resistance has become the main factor affecting the 
effectiveness of cancer treatment. ${ }^{34}$ Importantly, combination of resveratrol and chemotherapeutic drug can enhance the efficacy of chemotherapeutic drug treatment in cancer cells. It has been elucidated that resveratrol enhances the effectiveness of cisplatin and sensitizes cells to the cytotoxic effect of cisplatin in prostate cancer cells, ovarian cancer cells, non-small cell lung cancer cell lines, small cell lung cancer cells, hepatoma cells, colorectal cancer cells, breast cancer cells, and malignant mesothelioma cells. ${ }^{21,22,35-40}$ Rajesh and colleagues have demonstrated that resveratrol combined with low-dose docetaxel could improve clinical outcomes in clinical prostate cancer treatment. ${ }^{41}$ Resveratrol also can exert synergistic cytotoxic effect with docetaxel by blocking the HER-2-Akt signal axis in HER-2-overexpressing breast cancer cells. ${ }^{42}$

\section{Mechanism Of Resveratrol Inducing Autophagy}

Although a variety of signaling pathways for autophagy induced by resveratrol have been proposed, the mechanism of autophagy induced by resveratrol and which molecular pathways dictate the choice of resveratrol between autophagy and apoptosis in different cancer cells remain to be further studied. We first focus on mTOR, a serine/threonine kinase complex, as a target of resveratrol (Figure 1). mTOR, a central inhibitor of autophagy in mammalian cells, was first discovered as a target for the anti-fungal drug rapamycin in the early 1990s. ${ }^{43,44}$ mTOR exists in two different complex forms, mTOR complex 1 (mTORC1) and 2 (mTORC2). As a key factor in tumorigenesis, many cancer cells are accompanied by abnormal regulation of mTOR signaling pathway, which provides a basis for mTOR as a potential target for cancer therapy. ${ }^{45,46}$ It has been suggested by Liu and colleagues that resveratrol could enhance the interaction between mTOR and DEPTOR (DEP-domain containing mTOR-interacting protein), an inhibitor of mTOR. ${ }^{47,48}$ Activation of AMP-activated protein kinase (AMPK), an intracellular energy and metabolic receptor, is essential to regulate the energy and metabolic balance of the cell as a whole. It can act on a series of downstream target molecules through phosphorylation. ${ }^{49}$ Raptor, an integral part of the mTORC1 complex, is also one of the downstream target molecules of AMPK and can be phosphorylated by AMPK. Gwinn and colleagues have illustrated that resveratrol can repress mTORC1 activity by activating the endogenous AMPK. ${ }^{50}$ Dohyun Park and colleagues also demonstrated that resveratrol can inhibit mTOR directly, which is supported by the

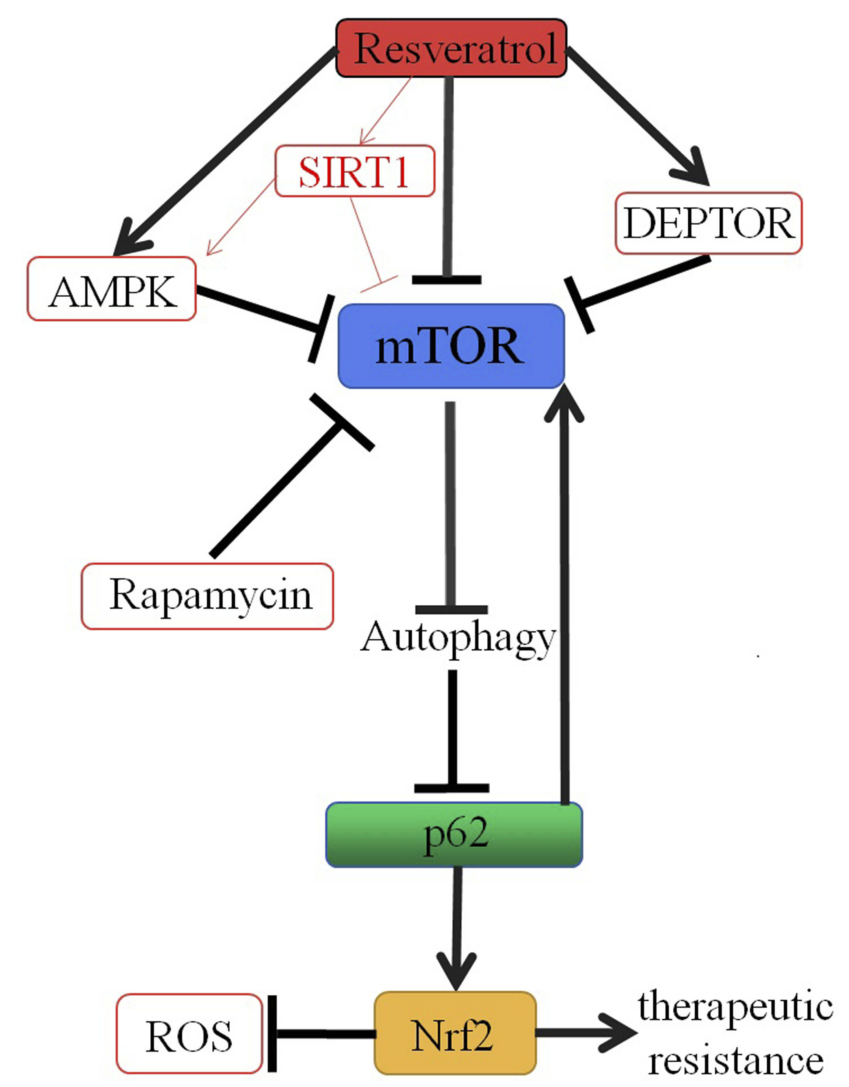

Figure I Mechanistic illustrations. Resveratrol induces autophagy by inhibiting mTOR through multiple pathways, such as promoting the association between mTOR and DEPTOR, triggering the phosphorylation of Raptor via activating AMPK and directly inhibiting mTOR, and as an SIRTI activator to active AMPK and directly inhibit mTOR. Autophagy accelerates the degradation of p62, and then, the mTOR activation is blocked and Nrf2 activation is suppressed. As a result, resveratrol can reverse multidrug resistance in cancer cells.

observation that the addition of resveratrol in vitro inhibits the activity of mTOR; additionally, this study provides that resveratrol has the ability to induce mTOR-dependent autophagy and reduce the viability of cancer cells. ${ }^{51}$ Resveratrol, also as an activator of SIRT1, induces protective autophagy via inhibiting Akt/mTOR and activating p38-MAPK in nonsmall cell lung cancer ${ }^{52}$ (Figure 1). Resveratrol has been shown to participate in both pro-survival and pro-death cellular process. However, the anticancer properties of resveratrol were probably due to the common effects of resveratrol on multiple signaling pathways, rather than just on a single pathway. The mechanism of resveratrol inducing autophagy against cancer remains to be further studied.

\section{Resveratrol Accelerates The Degradation Of $\mathbf{p 6 2}$}

p62, also called sequestosome 1 (SQSTM1), as a multidomain protein, contains different domains in the molecular 
structure. The sequence of p62 protein includes PB1 domain, ZZ zinc finger domain, TB domain, LC3-interaction region (LIR), Kelch-like ECH-associated protein 1 (Keap1)-interacting region (KIR), two nuclear localization signals (NLS1/2), one nuclear export signal (NES) motif, and near $\mathrm{C}$ terminal Ub-associated (UBA) domain. ${ }^{53}$ As a classical receptor of autophagy, p62 involves in selective autophagy through its PB1, LIR, and UBA domains. Under physiological conditions, the autophagy substrate protein interacts with the UBA domain of p62, and the substrate is loaded into the p62 oligomer to form the aggregation of p62 oligomer and autophagy substrate protein. Through the LIR region of p62, the aggregation is targeted into autophagosome and degrades in lysosome. ${ }^{54,55}$ The multi-functional structure of p62 makes it a multi-signal pathway center, which is involved in the maintenance and regulation of the basic function of cells. ${ }^{56}$

Importantly, it has been found that p62, as a target of autophagy (Figure 1), is constantly controlled by constitutive autophagy. ${ }^{57,58}$ Defects in autophagy upregulate p62. ${ }^{59}$ It was suggested that the abnormal accumulation of p62 due to impaired autophagy might promote tumorigenesis by activating the signaling pathway regulated by $\mathrm{p} 62 .{ }^{60}$ Importantly, high levels of p62 can lead to malignant transformation of epithelial cells, with or without impaired autophagy. ${ }^{61}$ Umemura and colleagues also suggested that the abnormal accumulation of p62 is a key pre-tumor event for the initiation of typical hepatocellular carcinomas (HCC) and can lead to activation of three important carcinogenesis factors: Nrf2, mTOR, and c-Myc. ${ }^{61}$ Resveratrol increases the degradation of p62 by enhancing autophagy, which in turn promotes the formation of apoptosis initiating Fas/Cav-1 complexes, and then the cleavage of beclin 1 and activation of caspase- 8 trigger apoptosis, thus enabling the transition from autophagy to apoptosis. ${ }^{62}$ Autophagy and apoptosis are both important biological phenomena involved in maintaining cell stability, structure, and function. Abnormalities of autophagy cause occurrence of cancer cells. We speculate that resveratrol, as an autophagy inducer, can prevent progression of oncogenic transformation as well as reduce recurrence of cancer via facilitating the degradation of $\mathrm{p} 62$.

\section{Resveratrol Inhibits The Downstream Signaling Pathways Of p62 \\ Resveratrol Inhibits mTOR}

mTOR is a central inhibitor of autophagy in mammalian cells. $^{63}$ As mentioned earlier, resveratrol can directly inhibit mTOR and then induce autophagy. mTOR also can be regulated by multiple signals, such as growth factors, hormones, and the levels of amino acid. When the levels of amino acid change, mTOR is activated by p62. The abnormal accumulation of p62 can be observed in different cancer cells, which may play a carcinogenic role by activating mTOR. ${ }^{64}$ Treatment with resveratrol, the activation of autophagy can reduce the accumulation of p $62,{ }^{62}$ following by blocking the mTOR activation, ${ }^{61}$ and further activates autophagy ${ }^{63}$ (Figure 1), which could enhance the anti-tumor effect of resveratrol. Whether there was a positive feedback and whether resveratrol could exert the maximum anti-tumor effect through this positive feedback remains to be further studied.

\section{Resveratrol Regulates Nrf2}

Nrf2 is a signal protein that regulates transcription and responds to oxidative stress. ${ }^{65}$ Intracellular Nrf2 abundance is strictly regulated by Keap1, a redox-sensitive E3 ubiquitin ligase substrate adaptor. ${ }^{66}$ Under homeostatic conditions, low levels of Nrf2 are primarily maintained by Keap1-mediated proteasomal degradation. ${ }^{67}$ Under oxidative stress conditions, Keap1 is oxidized at reactive cysteine residues, resulting in inactivation of Keap1 and stabilization of $\mathrm{Nrf2}$, which then translocates into the nucleus and subsequently binds to antioxidant response elements (ARE) of target genes. ${ }^{68,69}$ Komatsu and colleagues have shown that p62, as an endogenous protein, activates $\mathrm{Nrf} 2$ by competitive combination of Keap $1 .^{70}$ Jain and colleagues also demonstrated that $\mathrm{p} 62$ creates a positive feedback loop in the Keap1-Nrf2 pathway and the loop will be broken by the autophagic degradation of p62. ${ }^{71}$ When the autophagy is damaged, p62 accumulates and activates the Nrf2 by isolating the Keap1 into the inclusion bodies (Figure 1). Although the activation of Nrf2 has protective effect on the body during oxidative stress, the sustained activation of Nrf2 in cancer cells will improve the ability of anti-oxidation and detoxification of cancer cells and enhance therapeutic resistance of cancer cells. ${ }^{72}$ Recently, Daiha Shin and colleagues have shown that activation of the Nrf2/ARE pathway increases the resistance to glutathione peroxidase 4 (a regulator of ferroptosis) inhibitor in head and neck cancer cells (HNC) and that inhibition of this pathway reverses the resistance to ferroptosis (iron-dependent, non-apoptotic cell death) in HNC cells. ${ }^{73}$ Resveratrol, as an autophagy inducer, can facilitate p62 degradation, and as a result, p62-mediated $\mathrm{Nrf} 2$ activation is suppressed. It may be one of the 
pathways that resveratrol can reverse multidrug resistance in cancer cells (Figure 1). In addition, resveratrol can activate the Nrf2/ARE signaling pathway and play an antioxidant protective role by regulating the expression of antioxidant protein, oxidase, and phase II detoxifying enzymes, which is of great significance in the prevention of tumorigenesis. $^{74}$

\section{Resveratrol Inhibits The Proliferation Of Cancer Stem Cell (CSC)}

The CSC hypothesis suggests that cancer is the result of an unregulated expansion of the self-renewed CSC population. Understanding the self-renewal mechanism of CSCs will help identify molecular targets that are important for future treatments. ${ }^{75}$ The Wnt/ $\beta$-catenin pathway, associated with multiple cancers and with self-renewal of normal tissue cells, is a specifical therapeutic target. ${ }^{76,77}$ It has been verified that resveratrol induces autophagy in breast cancer stem-like cells (BCSCs) via suppressing Wnt/ $\beta$-catenin signaling pathway and significantly inhibits the proliferation of BCSCs. ${ }^{78} \mathrm{Chu}$ and colleagues demonstrated that attenuating $\mathrm{Wnt} / \beta$-catenin signaling enhances p62-mediated autophagy and apoptosis $>{ }^{79}$ Tian and colleagues also found that blocking the $\mathrm{Wnt} / \beta$-catenin signaling pathway could prevent Nrf2 signaling and enhance the efficacy of radiotherapy. ${ }^{80}$ In this review, we highlight that resveratrol, as an inducer of autophagy, can accelerate the degradation of p62 and inhibit the downstream signaling pathways of p62, such as mTOR and Nrf2 and reverse multidrug resistance in cancer cells. In the treatment of tumor stem cells, resveratrol has been shown to inhibit the Wnt/ $\beta$-catenin pathway, which in turn induces autophagy and inhibits the proliferation of stem cells, and it has been confirmed that there is a link between the Wnt/ $\beta$-catenin signaling pathway and p62 and Nrf2, but whether p62 and Nrf2 are involved in the mechanism of autophagy inducing by resveratrol via inhibiting the $\mathrm{Wnt} / \beta$-catenin in $\mathrm{CSC}$ needs to be confirmed by further research.

\section{Conclusion}

As mentioned earlier, resveratrol appears to be used both for cancer chemoprevention and for cancer treatment, which exhibits cytotoxicity and suppresses cell viability concentration-dependently, but has low toxicity in normal cells. Combined with different polyphenols, resveratrol elicits synergistic anticancer effects or antagonistic effects.
Resveratrol also enhances the effectiveness of chemotherapeutic drug and sensitizes cells to the cytotoxic effect. And in this review, we highlight that resveratrol, as an inducer of autophagy, reverses multidrug resistance in cancer cells by accelerating the degradation of p62 and inhibiting the downstream signaling pathways of p62, such as mTOR and Nrf2. And the combination of resveratrol with different polyphenols or chemotherapeutic drugs may produce synergistic anticancer effects and provide a promising novel anticancer treatment strategy.

Although it has been demonstrated that resveratrol can induce autophagy in several cancers, the mechanism of resveratrol inducing autophagy and the effect of resveratrol on autophagy and apoptosis in different cancers remain to be elucidated.

\section{Abbreviations}

mTOR, Mammalian Target of Rapamycin; Keap1, Kelchlike ECH-associated protein 1; Nrf2, Nuclear FactorErythroid 2-Related Factor 2; IARC, International Agency for Research on Cancer; ATG, autophagy-related gene; PI3K, phosphatidylinositol 3-kinase; TSC, tuberous sclerosis complex; ROS, reactive oxygen species; 3-MA, 3-methyladenine; DEPTOR, DEP-domain containing mTOR-interacting protein; AMPK, AMP-activated protein kinase; LIR, LC3-interaction region; KIR, Keap1-interacting region; NLS1/2, two nuclear localization signals; NES, one nuclear export signal; UBA, Ub-associated domain; HCC, hepatocellular carcinomas; ARE, antioxidant response elements; $\mathrm{HNC}$, head and neck cancer; CSC, cancer stem cell; BCSC, breast cancer stem-like cell.

\section{Acknowledgments}

This study was supported by grant from National Science Foundation of China (81670221 to YGZ).

\section{Author Contributions}

YYT and YGZ were involved in the conception of the study. YYT, YGZ, and DL were involved in the acquisition of the data and study design. YYT, WJS, and YGZ were involved in the writing of the article. YYT, YGZ, and LC critically revised the manuscript. All authors contributed to data analysis, drafting or revising the article, gave final approval of the version to be published, and agreed to be accountable for all aspects of the work.

\section{Disclosure}

The authors report no conflicts of interest in this work. 


\section{References}

1. Bray F. The evolving scale and profile of cancer worldwide: much ado about everything. Cancer Epidemiol Biomarkers Prev. 2016;25 (1):3-5. doi:10.1158/1055-9965.EPI-15-1109

2. Ko JH, Sethi G, Um JY, et al. The role of resveratrol in cancer therapy. Int J Mol Sci. 2017;18(12). doi:10.3390/ijms18122589

3. Kulkarni SS, Canto C. The molecular targets of resveratrol. Bba Mol Basis Dis. 2015;1852(6):1114-1123. doi:10.1016/j.bbadis.2014.10. 005

4. Kurgvietiene L, Staneviciene I, Mongirdiene A, Bernatoniene J. Multiplicity of effects and health benefits of resveratrol. Medicina Lithuania. 2016;52(3):148-155.

5. Chang $\mathrm{CH}$, Lee $\mathrm{CY}, \mathrm{Lu} \mathrm{CC}$, et al. Resveratrol-induced autophagy and apoptosis in cisplatin-resistant human oral cancer CAR cells: A key role of AMPK and Akt/mTOR signaling. Int J Oncol. 2017;50 (3):873-882. doi:10.3892/ijo.2017.3866

6. Bishop E, Bradshaw TD. Autophagy modulation: a prudent approach in cancer treatment? Cancer Chemoth Pharm. 2018;82(6):913-922. doi:10.1007/s00280-018-3669-6

7. Levine B, Kroemer G. Autophagy in the pathogenesis of disease. Cell. 2008;132(1):27-42. doi:10.1016/j.cell.2007.12.018

8. Levine B, Klionsky DJ. Development by self-digestion: molecular mechanisms and biological functions of autophagy. Dev Cell. 2004;6 (4):463-477.

9. Liang XH, Jackson S, Seaman M, et al. Induction of autophagy and inhibition of tumorigenesis by beclin 1. Nature. 1999;402(6762):672676. doi: $10.1038 / 45257$

10. Aita VM, Liang XH, Murty VV, et al. Cloning and genomic organization of beclin 1, a candidate tumor suppressor gene on chromosome 17q21. Genomics. 1999;59(1):59-65. doi:10.1006/geno.1999.5851

11. Choi AM, Ryter SW, Levine B. Autophagy in human health and disease. $N$ Engl J Med. 2013;368(7):651-662. doi:10.1056/NEJM ra1205406

12. Levine B. Cell biology - Autophagy and cancer. Nature. 2007;446 (7137):745-747. doi:10.1038/446745a

13. Mathew R, Karantza-Wadsworth V, White E. Role of autophagy in cancer. Nat Rev Cancer. 2007;7(12):961-967. doi:10.1038/nrc2254

14. Gozuacik D, Kimchi A. Autophagy as a cell death and tumor suppressor mechanism. Oncogene. 2004;23(16):2891-2906. doi:10.10 38/sj.onc. 1207521

15. Botti J, Djavaheri-Mergny M, Pilatte Y, Codogno P. Autophagy signaling and the cogwheels of cancer. Autophagy. 2006;2(2):67-73. doi:10.4161/auto.2.2.2458

16. Aggarwal BB, Bhardwaj A, Aggarwal RS, Seeram NP, Shishodia S, Takada Y. Role of resveratrol in prevention and therapy of cancer: preclinical and clinical studies. Anticancer Res. 2004;24(5A):27832840

17. Wang Y, Catana F, Yang Y, Roderick R, van Breemen RB. An LCMS method for analyzing total resveratrol in grape juice, cranberry juice, and in wine. J Agric Food Chem. 2002;50(3):431-435. doi:10. 1021/jf010812u

18. Huminiecki L, Horbanczuk J. The functional genomic studies of resveratrol in respect to its anti-cancer effects. Biotechnol Adv. 2018;36(6):1699-1708. doi:10.1016/j.biotechadv.2018.02.011

19. Jang M, Cai L, Udeani GO, et al. Cancer chemopreventive activity of resveratrol, a natural product derived from grapes. Science. 1997;275 (5297):218-220. doi:10.1126/science.275.5297.218

20. Levi F, Pasche C, Lucchini F, Ghidoni R, Ferraroni M, La Vecchia C. Resveratrol and breast cancer risk. Eur J Cancer Prev. 2005;14(2):139142.

21. Martinez-Martinez D, Soto A, Gil-Araujo B, Gallego B, Chiloeches A, Lasa M. Resveratrol promotes apoptosis through the induction of dual specificity phosphatase 1 and sensitizes prostate cancer cells to cisplatin. Food Chem Toxicol. 2019;124:273-279. doi:10.1016/j. fct.2018.12.014
22. Ma LJ, Li WP, Wang RX, et al. Resveratrol enhanced anticancer effects of cisplatin on non-small cell lung cancer cell lines by inducing mitochondrial dysfunction and cell apoptosis. Int J Oncol. 2015;47(4):1460-1468. doi:10.3892/ijo.2015.3124

23. Hua F, Shang S, Hu ZW. Seeking new anti-cancer agents from autophagy-regulating natural products. $J$ Asian Nat Prod Res. 2017;19(4):305-313. doi:10.1080/10286020.2017.1304385

24. Liu DC, He BZ, Lin LD, Malhotra A, Yuan NX. Potential of curcumin and resveratrol as biochemical and biophysical modulators during lung cancer in rats. Drug Chem Toxicol. 2019;42(3):328-334. doi:10.1080/01480545.2018.1523921

25. Alayev A, Berger SM, Holz MK. Resveratrol as a novel treatment for diseases with mTOR pathway hyperactivation. Resveratrol Health. 2015; 1348:116-123.

26. Talero E, Avila-Roman J, Motilva V. Chemoprevention with phytonutrients and microalgae products in chronic inflammation and colon cancer. Curr Pharm Design. 2012;18(26):3939-3965. doi:10.2174/ 138161212802083725

27. Du Q, Hu B, An HM, et al. Synergistic anticancer effects of curcumin and resveratrol in Hepa1-6 hepatocellular carcinoma cells. Oncol Rep. 2013;29(5):1851-1858. doi:10.3892/or.2013.2310

28. Tomas-Hernandez S, Blanco J, Rojas C, et al. Resveratrol potently counteracts quercetin starvation-induced autophagy and sensitizes HepG2 cancer cells to apoptosis. Mol Nutr Food Res. 2018;62(5). doi:10.1002/mnfr.201700879

29. Elmore S. Apoptosis: a review of programmed cell death. Toxicol Pathol. 2007;35(4):495-516. doi:10.1080/01926230701320 337

30. Maiuri MC, Zalckvar E, Kimchi A, Kroemer G. Self-eating and selfkilling: crosstalk between autophagy and apoptosis. Nat Rev Mol Cell Bio. 2007;8(9):741-752. doi:10.1038/nrm2239

31. Zhang J, Chiu JF, Zhang HW, et al. Autophagic cell death induced by resveratrol depends on the Ca2+/AMPK/mTOR pathway in A549 cells. Biochem Pharmacol. 2013;86(2):317-328. doi:10.1016/j.bcp. 2013.05.003

32. Lang FF, Qin ZY, Li F, et al. Apoptotic cell death induced by resveratrol is partially mediated by the autophagy pathway in human ovarian cancer cells. PLoS One. 2015;10(6). doi:10.1371/ journal.pone. 0129196

33. Wang HY, Peng Y, Wang J, et al. Effect of autophagy on the resveratrol-induced apoptosis of ovarian cancer SKOV3 cells. J Cell Biochem. 2019;120(5):7788-7793. doi:10.1002/jcb. 28053

34. Holohan C, Van Schaeybroeck S, Longley DB, Johnston PG. Cancer drug resistance: an evolving paradigm. Nat Rev Cancer. 2013;13 (10):714-726. doi:10.1038/nrc3599

35. Lee YJ, Lee GJ, Yi SS, et al. Cisplatin and resveratrol induce apoptosis and autophagy following oxidative stress in malignant mesothelioma cells. Food Chem Toxicol. 2016;97:96-107. doi:10.1016/j.fct.2016.08.033.

36. Nessa MU, Beale P, Chan C, Yu JQ, Huq F. Combinations of resveratrol, cisplatin and oxaliplatin applied to human ovarian cancer cells. Anticancer Res. 2012;32(1):53-59.

37. Li WP, Shi Y, Wang RX, Pan L, Ma LJ, Jin FG. Resveratrol promotes the sensitivity of small-cell lung cancer H446 cells to cisplatin by regulating intrinsic apoptosis. Int J Oncol. 2018;53(5):2123-2130. doi:10.3892/ijo.2018.4533

38. Liu ZY, Peng Q, Li Y, Gao Y. Resveratrol enhances cisplatin-induced apoptosis in human hepatoma cells via glutamine metabolism inhibition. BMB Rep. 2018;51(9):474-479.

39. Osman AMM, Al-Malki HS, Al-Harthi SE, El-Hanafy AA, Elashmaoui HM, Elshal MF. Modulatory role of resveratrol on cytotoxic activity of cisplatin, sensitization and modification of cisplatin resistance in colorectal cancer cells. Mol Med Rep. 2015;12(1):13681374. doi: $10.3892 / \mathrm{mmr} .2015 .3513$ 
40. Leon-Galicia I, Diaz-Chavez J, Albino-Sanchez ME, et al. Resveratrol decreases Rad51 expression and sensitizes cisplatin-resistant MCF-7 breast cancer cells. Oncol Rep. 2018;39(6):3025-3033. doi:10.3892/or.2018.6336

41. Singh SK, Banerjee S, Acosta EP, Lillard JW, Singh R. Resveratrol induces cell cycle arrest and apoptosis with docetaxel in prostate cancer cells via a p53/p21(WAF1/CIP1) and p27(KIP1) pathway. Oncotarget. 2017;8(10):17216-17228. doi:10.18632/oncotarget.153 03

42. Vinod BS, Nair HH, Vijayakurup V, et al. Resveratrol chemosensitizes HER-2-overexpressing breast cancer cells to docetaxel chemoresistance by inhibiting docetaxel-mediated activation of HER-2-Akt axis. Cell Death Discovery. 2015;1. doi:10.1038/ cddiscovery.2015.61

43. Brown EJ, Albers MW, Shin TB, et al. A mammalian protein targeted by G1-arresting rapamycin-receptor complex. Nature. 1994;369 (6483):756-758. doi:10.1038/369756a0

44. Sabatini DM, Erdjument-Bromage H, Lui M, Tempst P, Snyder SH. RAFT1: a mammalian protein that binds to FKBP12 in a rapamycindependent fashion and is homologous to yeast TORs. Cell. 1994;78 (1):35-43. doi:10.1016/0092-8674(94)90570-3

45. He X, Wang Y, Zhu JH, Orloff M, Eng C. Resveratrol enhances the anti-tumor activity of the mTOR inhibitor rapamycin in multiple breast cancer cell lines mainly by suppressing rapamycin-induced AKT signaling. Cancer Lett. 2011;301(2):168-176. doi:10.1016/j. canlet.2010.11.012

46. Rogers-Broadway KR, Kumar J, Sisu C, et al. Differential expression of mTOR components in endometriosis and ovarian cancer: effects of rapalogues and dual kinase inhibitors on mTORC1 and mTORC2 stoichiometry. Int J Mol Med. 2019;43(1):47-56. doi:10.3892/ ijmm.2018.3967

47. Peterson TR, Laplante M, Thoreen CC, et al. DEPTOR is an mTOR inhibitor frequently overexpressed in multiple myeloma cells and required for their survival. Cell. 2009;137(5):873-886. doi:10.1016/ j.cell.2009.03.046

48. Liu ML, Wilk SA, Wang AP, et al. Resveratrol inhibits mTOR signaling by promoting the interaction between mTOR and DEPTOR. J Biol Chem. 2010;285(47):36387-36394. doi:10.1074/ jbc.M110.169284

49. Thirupathi ACY. Role of AMPK and its molecular intermediates in subjugating cancer survival mechanism. Life Sci. 2019. doi:10.1016/j. lfs.2019.04.039

50. Gwinn DM, Shackelford DB, Egan DF, et al. AMPK phosphorylation of raptor mediates a metabolic checkpoint. Mol Cell. 2008;30 (2):214-226. doi:10.1016/j.molcel.2008.03.003

51. Park D, Jeong H, Lee MN, et al. Resveratrol induces autophagy by directly inhibiting mTOR through ATP competition. Sci Rep. 2016;6:21772. doi:10.1038/srep21772

52. Wang J, Li JM, Cao NQ, Li Z, Han JY, Li L. Resveratrol, an activator of SIRT1, induces protective autophagy in non-small-cell lung cancer via inhibiting Akt $/ \mathrm{mTOR}$ and activating $\mathrm{p} 38$-MAPK. Oncotargets Ther. 2018;11:7777-7785. doi:10.2147/OTT.S159095

53. Komatsu M, Kageyama S, Ichimura Y. p62/SQSTM1/A170: physiology and pathology. Pharmacol Res. 2012;66(6):457-462. doi:10.1016/j.phrs.2012.07.004

54. Pankiv S, Clausen TH, Lamark T, et al. p62/SQSTM1 binds directly to $\mathrm{Atg} 8 / \mathrm{LC} 3$ to facilitate degradation of ubiquitinated protein aggregates by autophagy. J Biol Chem. 2007;282(33):24131-24145. doi:10.1074/jbc.M702824200

55. Wooten MW, Geetha T, Babu JR, et al. Essential role of sequestosome 1/p62 in regulating accumulation of Lys63-ubiquitinated proteins. J Biol Chem. 2008;283(11):6783-6789. doi:10.1074/jbc.M70 9496200

56. Moscat J, Diaz-Meco MT. p62: a versatile multitasker takes on cancer. Trends Biochem Sci. 2012;37(6):230-236. doi:10.1016/j. tibs.2012.02.008
57. Komatsu M, Waguri S, Koike M, et al. Homeostatic levels of p62 control cytoplasmic inclusion body formation in autophagy-deficient mice. Cell. 2007;131(6):1149-1163. doi:10.1016/j.cell.2007.10.035

58. Bjorkoy G, Lamark T, Brech A, et al. p62/SQSTM1 forms protein aggregates degraded by autophagy and has a protective effect on huntingtin-induced cell death. J Cell Biol. 2005;171(4):603-614. doi:10.1083/jcb.200507002

59. Lan SH, Wu SY, Zuchini R, et al. Autophagy suppresses tumorigenesis of hepatitis B virus-associated hepatocellular carcinoma through degradation of microRNA-224. Hepatology. 2014;59(2):505-517. doi:10.1002/hep.26659

60. Mathew R, Karp CM, Beaudoin B, et al. Autophagy suppresses tumorigenesis through elimination of p62. Cell. 2009;137(6):10621075. doi:10.1016/j.cell.2009.03.048

61. Umemura A, He F, Taniguchi $\mathrm{K}$, et al. p62, upregulated during preneoplasia, induces hepatocellular carcinogenesis by maintaining survival of stressed HCC-initiating cells. Cancer Cell. 2016;29 (6):935-948. doi:10.1016/j.ccell.2016.04.006

62. Zhang J, Ma K, Qi TT, et al. P62 Regulates resveratrol-mediated Fas/ Cav-1 complex formation and transition from autophagy to apoptosis. Oncotarget. 2015;6(2):789-801. doi:10.18632/oncotarget.2733

63. Lum JJ, DeBerardinis RJ, Thompson CB. Autophagy in metazoans: cell survival in the land of plenty. Nat Rev Mol Cell Bio. 2005;6 (6):439-448. doi:10.1038/nrm1660

64. Saxton RA, Sabatini DM. mTOR signaling in growth, metabolism, and disease. Cell. 2017;168(6):960-976. doi:10.1016/j.cell.2017.02. 004

65. Ma Q. Role of Nrf2 in oxidative stress and toxicity. Annu Rev Pharmacol. 2013;53:401-+. doi:10.1146/annurev-pharmtox-011112140320

66. Itoh K, Wakabayashi N, Katoh Y, et al. Keap1 represses nuclear activation of antioxidant responsive elements by Nrf2 through binding to the amino-terminal Neh2 domain. Genes Dev. 1999;13(1):7686. doi: $10.1101 / \mathrm{gad} .13 .1 .76$

67. Zhang DD. Mechanistic studies of the Nrf2-Keap1 signaling pathway. Drug Metab Rev. 2006;38(4):769-789. doi:10.1080/036025 30600971974

68. Baird L, Swift S, Lleres D, Dinkova-Kostova AT. Monitoring Keap1Nrf2 interactions in single live cells. Biotechnol Adv. 2014;32 (6):1133-1144. doi:10.1016/j.biotechadv.2014.03.004

69. Dinkova-Kostova AT, Holtzclaw WD, Cole RN, et al. Direct evidence that sulfhydryl groups of Keap1 are the sensors regulating induction of phase 2 enzymes that protect against carcinogens and oxidants. Proc Natl Acad Sci U S A. 2002;99(18):11908-11913. doi:10.1073/pnas.172398899

70. Komatsu M, Kurokawa H, Waguri S, et al. The selective autophagy substrate p62 activates the stress responsive transcription factor Nrf2 through inactivation of Keap1. Nat Cell Biol. 2010;12(3):213-U217. doi: $10.1038 /$ ncb2021

71. Jain A, Lamark T, Sjottem E, et al. p62/SQSTM1 is a target gene for transcription factor NRF2 and creates a positive feedback loop by inducing antioxidant response element-driven gene transcription. $J$ Biol Chem. 2010;285(29):22576-22591. doi:10.1074/jbc.M110.11 8976

72. Kitamura H, Motohashi H. NRF2 addiction in cancer cells. Cancer Sci. 2018;109(4):900-911. doi:10.1111/cas.13537

73. Shin D, Kim EH, Lee J, Roh JL. Nrf2 inhibition reverses resistance to GPX4 inhibitor-induced ferroptosis in head and neck cancer. Free Radical Bio Med. 2018;129:454-462. doi:10.1016/j.freeradbiomed. 2018.10.426

74. Whitlock NC, Baek SJ. The anticancer effects of resveratrol: modulation of transcription factors. Nutr Cancer Int J. 2012;64(4):493502. doi:10.1080/01635581.2012.667862

75. Lobo NA, Shimono Y, Qian D, Clarke MF. The biology of cancer stem cells. Annu Rev Cell Dev Bi. 2007;23:675-699. doi:10.1146/ annurev.cellbio.22.010305.104154 
76. Taipale J, Beachy PA. The Hedgehog and Wnt signaling pathways in cancer. Nature. 2001;411(6835):349-354. doi:10.1038/ 35077219

77. de Lau W, Barker N, Clevers H. WNT signaling in the normal intestine and colorectal cancer. Front Biosci Landmrk. 2007;12:471-491. doi: $10.2741 / 2076$

78. Fu YJ, Chang H, Peng XL, et al. Resveratrol inhibits breast cancer stem-like cells and induces autophagy via suppressing Wnt/betacatenin signaling pathway. PLoS One. 2014;9(7):e102535.

79. Chu CW, Ko HJ, Chou $\mathrm{CH}$, et al. Thioridazine enhances P62mediated autophagy and apoptosis through Wnt/-catenin signaling pathway in glioma cells. Int J Mol Sci. 2019;20(3). doi:10.3390/ ijms20030473

80. Tian D, Shi Y, Chen DX, Liu Q, Fan FY. The Wnt inhibitor LGK974 enhances radiosensitivity of HepG2 cells by modulating Nrf2 signaling. Int J Oncol. 2017;51(2):545-554. doi:10.3892/ijo.2017. 4042
81. Zhong LX, Zhang Y, Wu ML, et al. Resveratrol and STAT inhibitor enhance autophagy in ovarian cancer cells. Cell Death Discovery. 2016;2. doi:10.1038/cddiscovery.2016.9

82. Miki H, Uehara N, Kimura A, et al. Resveratrol induces apoptosis via ROS-triggered autophagy in human colon cancer cells. Int J Oncol. 2012;40(4):1020-1028. doi:10.3892/ijo.2012.1325

83. Selvaraj S, Sun YY, Sukumaran P, Singh BB. Resveratrol activates autophagic cell death in prostate cancer cells via downregulation of STIM1 and the mTOR pathway. Mol Carcinogen. 2016;55(5):818831. doi:10.1002/mc.22324

84. Alayev A, Berger SM, Kramer MY, Schwartz NS, Holz MK. The combination of rapamycin and resveratrol blocks autophagy and induces apoptosis in breast cancer cells. J Cell Biochem. 2015;116 (3):450-457. doi:10.1002/jcb.24997

\section{Publish your work in this journal}

OncoTargets and Therapy is an international, peer-reviewed, open access journal focusing on the pathological basis of all cancers, potential targets for therapy and treatment protocols employed to improve the management of cancer patients. The journal also focuses on the impact of management programs and new therapeutic agents and protocols on patient perspectives such as quality of life, adherence and satisfaction. The manuscript management system is completely online and includes a very quick and fair peer-review system, which is all easy to use. Visit http://www.dovepress.com/ testimonials.php to read real quotes from published authors. 